\section{Associations of serum soluble Fas and Fas ligand (FasL) with outcomes in systemic lupus erythematosus}

\author{
Fabien B Vincent (10 , ${ }^{1}$ Rangi Kandane-Rathnayake, ${ }^{1}$ Rachel Koelmeyer, ${ }^{1}$ \\ James Harris, ${ }^{1}$ Alberta Y Hoi (D) , ${ }^{1}$ Fabienne Mackay, ${ }^{2,3}$ Eric F Morand ${ }^{1}$
}

To cite: Vincent FB, Kandane-Rathnayake $\mathrm{R}$, Koelmeyer R, et al. Associations of serum soluble Fas and Fas ligand (FasL) with outcomes in systemic lupus erythematosus. Lupus Science \& Medicine 2020;7:e000375. doi:10.1136/ lupus-2019-000375

Received 18 December 2019 Revised 24 April 2020 Accepted 13 May 2020

\section{Check for updates}

(C) Author(s) (or their employer(s)) 2020. Re-use permitted under CC BY-NC. No commercial re-use. See rights and permissions. Published by BMJ.

${ }^{1}$ Centre for Inflammatory Diseases, School of Clinical Sciences at Monash Health, Monash University, Clayton, Victoria, Australia

${ }^{2}$ Department of Immunology and Pathology, Monash University, Central Clinical School, Alfred Medical Research and Education Precinct (AMREP), Melbourne,

Victoria, Australia

${ }^{3}$ Department of Microbiology and Immunology, School of Biomedical Sciences, Faculty of Medicine, Dentistry and Health Sciences, The University of Melbourne, Parkville, Victoria, Australia

Correspondence to

Professor Eric F Morand; eric. morand@monash.edu

\section{ABSTRACT}

Objective Fas/Fas ligand (FasL) and B cell-activating factor (BAFF) signalling have pivotal roles in SLE pathogenesis. We investigated the clinical associations of serum concentrations of soluble Fas (sFas) and soluble FasL (SFasL) in SLE and their relationship with BAFF. Methods Serum sFas and sFasL were quantified by multiplex assay, and BAFF by ELISA, in 118 patients with SLE and 17 healthy controls (HC). SLE disease activity and organ damage were assessed using the Systemic Lupus Erythematosus Disease Activity Index 2000 (SLEDAl-2K) and the Systemic Lupus International Collaborating Clinics Damage Index.

Results sFas, sFasL and BAFF were detectable in all samples. Serum sFas and sFasL were significantly higher in SLE compared with HC. In univariable regression analyses, patients with active renal disease and those with flare had significantly higher levels of sFas compared with those without. High serum BAFF in patients with SLE was associated with increased sFas but not sFasL. The association between sFas and renal disease remained significant after adjusting for BAFF, but the association with flare attenuated. High sFas levels were associated with increased time-adjusted mean SLEDAI-2K, even after adjusting for BAFF, and with higher odds of flare over time. In contrast, high sFasL was associated with reduced organ damage over time. Serum sFasL/sFas ratio was negatively associated with active overall disease, flare and organ damage.

Conclusions Serum sFas is associated with active renal SLE, and active disease and flare over time, while sFasL/ sFas ratio is negatively associated with disease activity and organ damage accrual. Treatments correcting abnormal levels of sFas/FasL may be worthy of evaluation in SLE.

\section{INTRODUCTION}

SLE is an idiopathic heterogeneous chronic systemic autoimmune disease. ${ }^{1}$ Among many implicated cytokine systems, evidence suggests that the tumour necrosis factor (TNF) superfamily ligand Fas ligand (FasL), which signals through Fas, may play a role in SLE pathogenesis. ${ }^{2}$ Signalling by FasL through Fas leads to apoptosis, ${ }^{3}$ and impaired activation-induced cell death induced by mutations in mouse genes encoding for Fas (lpr) and FasL ( $g l d)$ leads to spontaneous mouse models reminiscent of human SLE. ${ }^{4-6}$ The soluble forms of Fas (sFas) and FasL (sFasL) both act mainly as decoys for the Fas-mediated apoptosis pathway, ${ }^{7-11}$ potentially leading to increased survival of Fas-bearing target cells. However, sFasL mediates proinflammatory effects through Fas, ${ }^{12-14}$ while a proapoptotic function of oligomeric, but not monomeric, sFas has been described, ${ }^{15}$ indicating potentially divergent functions of sFasL and sFas that are especially relevant when sFas is abundant, such as in autoimmune disease. ${ }^{1516}$

Compared with healthy controls (HC), serum sFas concentrations are reported in most studies to be elevated in patients with SLE. ${ }^{10}{ }^{17-25}$ However, reported findings are inconsistent in regard to the relationship between serum sFas and disease activity, in that a positive relationship is shown in some studies, ${ }^{1718222526}$ but not in others. ${ }^{16} 1924$ Only a very few studies have investigated serum $\mathrm{sFas}$ as a biomarker for specific organ activity, particularly associated with renal, neurological and mucocutaneous SLE. ${ }^{16} 18202728$ Fewer studies have focused on sFasL in SLE, although it has been reported to be elevated compared with HC in most studies. ${ }^{21} 232629$ A positive relationship between sFasL and SLE disease activity has been reported in one study, ${ }^{26}$ but not in another. ${ }^{30}$

In light of these discrepancies, measuring the ratio between sFas and sFasL may be of interest, since both proteins belong to the same immunological pathway and are able to modulate the Fas/FasL system. ${ }^{21}$ The use of this ratio may better reflect the Fas/FasL system biological activity as a whole, where sFas and sFasL may have divergent biological functions. ${ }^{21}{ }^{31}$ While the ratio has been investigated in other conditions, ${ }^{32-35}$ only one prior study in a small cohort of 15 patients assessed this ratio in relation to SLE disease activity. ${ }^{21}$ 
B cell-activating factor from the TNF family (BAFF) is a key pathogenic cytokine in SLE. ${ }^{36}$ The Fas/FasL disrupted MRL-lpr/lpr lupus-prone mouse model is characterised by high serum BAFF concentrations, ${ }^{37} 38$ and our group previously described an interaction between the BAFF system and Fas/FasL system-mediated marginal zone B cell apoptosis in mice ${ }^{39}$ Since BAFF potentially plays a role in the regulation of the Fas/FasL apoptotic pathway, ${ }^{39}$ measuring the key components of both BAFF and Fas/FasL systems is an interest in human SLE. To date, only one study has measured both BAFF and components of the Fas/FasL system in their soluble forms, within a larger pool of 52 soluble factors. ${ }^{40}$

Here, we aimed to investigate the clinical associations of serum sFas and sFasL as well as the ratio of these molecules in an SLE cohort larger and better characterised than any previously described, and investigate whether associations with SLE are independent of serum BAFF concentrations. We also assessed the associations of baseline measurements of these factors with clinical progression over time.

\section{MATERIALS AND METHODS}

\section{Participants and clinical assessments}

Adult patients fulfilling the 1997 American College of Rheumatology revised criteria for SLE classification were enrolled at the Monash Lupus Clinic (Clayton, Victoria, Australia) between December 2009 and July 2014, as previously described. ${ }^{41}$ Demographic and clinical data, including disease duration, activity and damage, routine laboratory markers, and treatment, were recorded at baseline and routine visits. Overall disease activity was assessed using a hybrid Systemic Lupus Erythematosus Disease Activity Index 2000 (SLEDAI-2K). ${ }^{42}$ Disease activity at a given visit/sample date was classified as inactive if SLEDAI-2K was $\leq 4$, using the SLEDAI-2K threshold used in the definition of the lupus low disease activity state $^{43}$; an SLEDAI-2K $>4$ was thus classified as active. To assess individual organ-specific disease activity, components of the SLEDAI-2K scoring system were grouped into nine organ domains. Organ-specific disease activity was defined as activity in at least one component of the SLEDAI-2K that pertains to an organ domain. ${ }^{44}$ Average disease activity over time was assessed using the timeadjusted mean SLEDAI-2K (AMS) ${ }^{45} 46$ and active disease over time defined as AMS $>4 .{ }^{47}$ Flare was assessed using the Safety of Estrogens in Lupus Erythematosus National Assessment Flare Index. ${ }^{48}$ Patients were classified as having irreversible organ damage using the Systemic Lupus International Collaborating Clinics/American College of Rheumatology Damage Index, as previously described. ${ }^{49}$ Cut-off values for routine laboratory markers analysis were as follows: high $\mathrm{C}$ reactive protein (CRP) $(>3 \mathrm{mg} / \mathrm{L})$, high erythrocyte sedimentation rate (ESR) ( $\geq 25 \mathrm{~mm} /$ hour), proteinuria (urine protein to creatinine ratio (UPCR) $>0.05 \mathrm{~g} / \mathrm{mmol})$, low C3 $(<0.79 \mathrm{~g} / \mathrm{L})$ and low C4 $(<0.16 \mathrm{~g} / \mathrm{L})$. All patients received standard-of-care therapy. Between June and August 2014, healthy individuals were enrolled as an HC group. All individuals gave written informed consent.

\section{Serum cytokines and soluble receptor quantification}

Whole blood was collected by venepuncture in association with routine clinical visits. Serum was isolated using serum-separating collection tube, as previously described,$^{50}$ and stored at $-80^{\circ} \mathrm{C}$, until further use. Serum sFas and sFasL concentrations were quantified using a commercial Luminex screening assay (polystyrene beads; Cat \#LXSAH, R\&D Systems, Minneapolis, Minneapolis, USA), using a Bio-Rad Bio-Plex 200 system, following the manufacturer's protocol. Serum BAFF concentrations were quantified using a commercial ELISA kit (Quantikine, Cat \#SBLYS0B, R\&D Systems), following the manufacturer's protocol. Analysis of serum BAFF in a smaller cohort of patients $(n=87)$ that are included in this larger sample has been reported separately. ${ }^{51}$

\section{Statistical analysis}

Statistical analysis was performed using Stata V.14.2 (StataCorp, College Station, Texas, USA) software. Continuous data were described as median (IQR) or mean (SD) according to data distribution. Categorical data were summarised as number (frequency). Correlations between variables were examined using Spearman's rank or Pearson's correlation test, depending on data distribution. We compared some demographics between patients with SLE and HC using t-test to compare means, and Pearson's $\chi^{2}$ test or Fisher's exact test to compare proportions. Linear regression was used to examine associations of serum sFas and sFasL concentrations, and their ratio, with clinical outcomes in cross-sectional analysis. Serum BAFF, sFas and sFasL concentrations were $\log _{10}$-transformed before including in the linear regression models. In the occurrence of non-normal distribution after data transformation, a bootstrap method with 50-sample derivation was incorporated to derive robust CI. Results were shown as geometric mean (GM) (antilogs of the means derived from linear regressions) and the ratios of GM (antilogs of regression coefficient) with corresponding 95\% CI. Logistic regression analysis was used to examine the associations of baseline serum cytokines and soluble receptor concentrations with longitudinal clinical outcomes. Based on the medians, serum BAFF, Fas and sFasL concentrations were grouped as low ( $\leq$ median) and high (>median) subsets to perform logistic regression analysis. Variables which demonstrated univariable associations with $\mathrm{p}<0.1$ with both independent (exposure) and dependent (outcome) factors were included in multivariable linear and logistic regression models as potential confounders. $\mathrm{P}<0.05$ was considered statistically significant.

\section{Patient and public involvement}

Patients or the public were not involved in the design, or conduct, or reporting, or dissemination plans of our research. 


\section{RESULTS}

\section{Participant characteristics}

One hundred and eighteen patients with SLE were included in this study. Patients' characteristics are summarised in table 1. Briefly, the mean (SD) age was 45.4 (14.2) years and the median (IQR) disease duration was 7.2 (3.914.7 ) years. Of the patients, $85 \%$ were female, $48 \%$ were of Asian ethnicity, 33\% had active disease and $57 \%$ were receiving glucocorticoids. Follow-up clinical data were available for a median (IQR) of $2.1(1.8-2.6)$ years after serum sampling. The HC cohort comprised 17 individuals, among whom $88 \%$ were female and nearly $30 \%$ were of Asian ethnicity. The median (IQR) age was 41 (28-44) years. The HC cohort was gender-matched and ethnicitymatched to the SLE cohort, but was significantly younger (mean (SD) 37.4 (10.2) vs 45.4 (14.2) years; p=0.03). Hence, age was included as a potential confounder in the multivariable regression analysis models when comparing SLE with HC cohorts.

\section{Cytokine and soluble receptor concentrations in SLE and HC}

sFas and sFasL were detectable in all serum SLE and HC samples. Serum sFas concentration was significantly increased in SLE compared with HC (ratio of GM 1.44; 95\% CI 1.19 to $1.74 ; \mathrm{p}<0.01)$, and after adjusting for age (ratio of GM 1.4; 95\% CI 1.15 to $1.7 ; \mathrm{p}<0.01$ ) (figure $1 \mathrm{~A}$ ). Serum sFasL concentrations were also significantly increased in SLE when compared with HC (ratio of GM 1.18; 95\% CI 1.01 to $1.37 ; \mathrm{p}=0.04$ ), confirmed after adjusting for age (ratio of GM 1.2; 95\% CI 1.01 to 1.42; $\mathrm{p}=0.04$ ) (figure $1 \mathrm{~B}$ ). Serum $\mathrm{sFasL} / \mathrm{sFas}$ ratio was significantly decreased in SLE (ratio of GM 0.82; $95 \%$ CI 0.68 to 0.99 ; $\mathrm{p}=0.04$ ); however, this association was not confirmed after adjusting for age (ratio of GM 0.86; $95 \%$ CI 0.64 to $1.15 ; \mathrm{p}=0.31$ ) (figure 1C). Serum sFas and sFasL concentrations were not correlated with each other in either the SLE or HC cohort (figure 2A,B).

Serum BAFF was detectable in all SLE and HC serum samples. Serum BAFF was significantly increased in SLE compared with HC (ratio of GM 1.15; 95\% CI 1.02 to 1.3; $\mathrm{p}=0.03$ ), and after adjusting for age (ratio of GM 1.16; 95\% CI 1.02 to $1.3 ; \mathrm{p}=0.02$ ) (figure 1D). Serum sFas was weakly positively correlated with BAFF concentration in SLE ( $\mathrm{r}=0.22 ; \mathrm{p}=0.02$ ), but not in HC (figure 2C,D). Linear regression analysis confirmed a positive association between sFas and BAFF (ratio of GM 1.2; 95\% CI 1.04 to $1.38 ; \mathrm{p}=0.01$ ). No significant correlation was observed between serum concentrations of BAFF and sFasL or sFasL/sFas ratio in SLE or HC samples (figure 2E-H).

\section{Clinical associations: cross-sectional analysis at time of sampling}

We investigated whether serum concentrations were associated with clinical parameters at the time of sampling, using linear regression, adjusting for potential confounders including serum BAFF. Serum sFas was significantly higher in patients with active renal disease, defined as renal SLEDAI-2K $>0$ or by the presence of proteinuria
Table 1 Demographic, clinical and biological characteristics of the SLE cohort

\begin{tabular}{ll}
\hline Characteristics & SLE cohort \\
\hline $\mathbf{( n = 1 1 8 )}$
\end{tabular}

Data are expressed as mean (SD), median (IQR) or as number (percentage). *Other ethnicities included Hispanic (1; 0.8\%), Maori $(2 ; 1.7 \%)$ and Samoan $(1 ; 0.8 \%)$.

tIndividual organ domain disease activity was assessed by the SLEDAI-2K. $\ddagger$ Encompasses mild, moderate and severe flares.

§Immunosuppressants include methotrexate, azathioprine, mycophenolate mofetil, mycophenolate acid, leflunomide, ciclosporin A and/or cyclophosphamide.

IProteinuria defined as UPCR $>0.05 \mathrm{~g} / \mathrm{mmol}$, using SLEDAl-2K cut-off. AMS, adjusted mean SLEDAI-2K; CRP, C reactive protein; dsDNA, doublestranded DNA; ESR, erythrocyte sedimentation rate; SDI, Systemic Lupus International Collaborating Clinics/American College of Rheumatology Damage Index; SLEDAI-2K, Systemic Lupus Erythematosus Disease Activity Index 2000; UPCR, urine protein to creatinine ratio.

(table 2). Similarly, serum sFas was significantly higher in patients with flare (table 2). The association between 
A

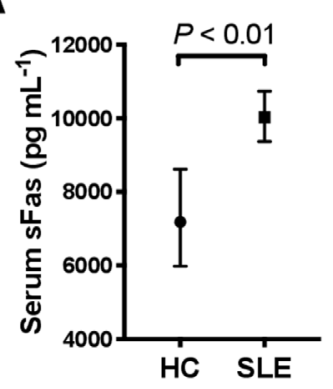

C

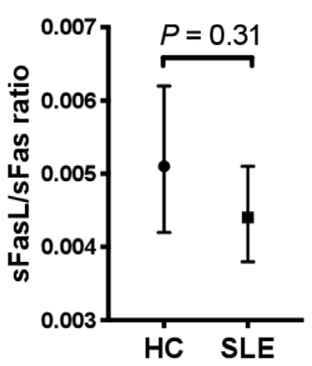

B

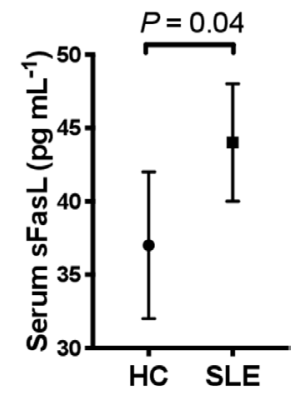

D

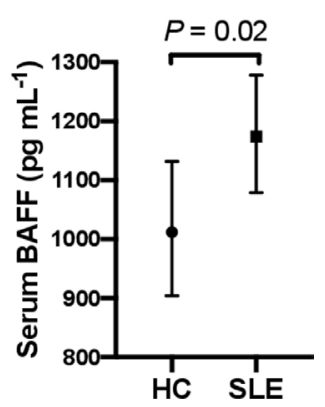

Figure 1 Serum sFas, sFasL, sFasL/sFas ratio and BAFF in SLE. Age-adjusted geometric mean derived using multivariable linear regression analysis of serum sFas concentrations (A), sFasL concentrations (B), sFasL/sFas ratio (C), and BAFF concentrations (D) in $\mathrm{HC}(n=17)$ and in patients with SLE $(n=118)$. BAFF, B cell-activating factor from the tumour necrosis factor family; $\mathrm{HC}$, healthy control; sFas, soluble Fas; sFasL, soluble Fas ligand; SLE, systemic lupus erythematosus.

serum sFas and active renal SLE was confirmed after adjusting for serum BAFF (ratio of GM 1.35; 95\% CI 1.13 to $1.62 ; \mathrm{p}<0.01$ ), but the association with flare attenuated (ratio of GM 1.13; $95 \%$ CI 0.96 to $1.33 ; \mathrm{p}=0.15$ ). We did not observe any association between serum sFas and overall disease activity or other organ-specific SLE disease activity, organ damage or laboratory markers (table 2).

In contrast, serum sFasL levels were reduced in patients with flare (table 2). This association was, however, attenuated after adjusting for use of immunosuppressants (ratio of GM 0.86 ; $95 \%$ CI 0.68 to $1.09 ; \mathrm{p}=0.21$ ). Serum sFasL was also significantly decreased in patients with high ESR and in those receiving glucocorticoids (table 2). No statistically significant association emerged of sFasL with disease activity, organ damage or other laboratory markers (table 2).

The sFasL/sFas ratio was negatively associated with active overall disease activity, and with renal and mucocutaneous disease activity, in univariable analysis (table 2). The association between sFasL/sFas ratio and overall disease activity was confirmed after adjusting for use of immunosuppressants (ratio of GM 0.79; 95\% CI 0.65 to 0.96 ; $\mathrm{p}=0.02$ ), but the association between $\mathrm{sFasL} / \mathrm{sFas}$ ratio and active renal and mucocutaneous disease was attenuated (renal: adjusted for use of immunosuppressants, ratio of

A

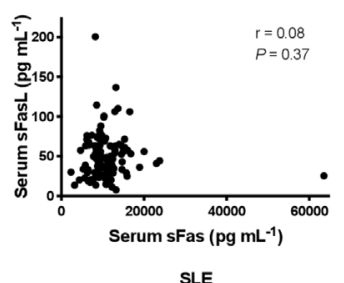

C

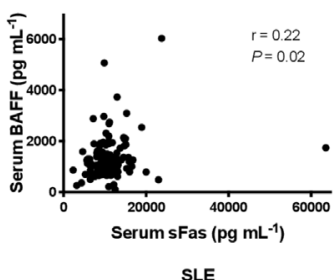

E

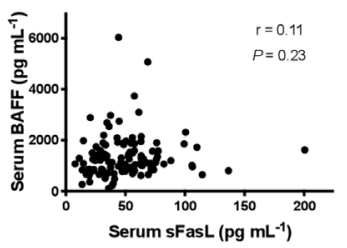

SLE

G

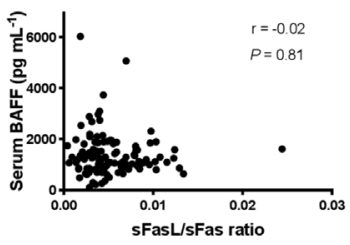

SLE
B

$\mathrm{HC}$

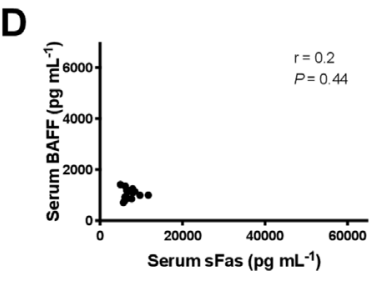

HC

$\mathbf{F}$

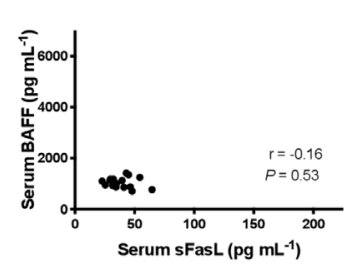

HC

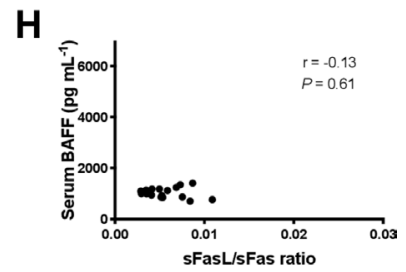

HC

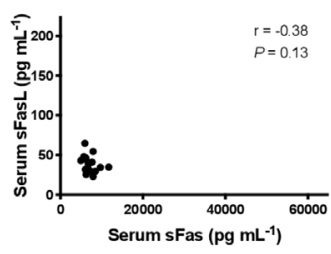

Figure 2 Correlation between serum sFas, sFasL, sFasL/ sFas ratio, and BAFF concentrations in SLE and HC. (A-B) Correlation between serum sFas and SFasL concentrations in (A) SLE $(n=118)$ and (B) HC ( $n=17)$. (C-D) Correlation between serum sFas and BAFF concentrations in (C) SLE $(n=118)$ and (D) HC $(n=17)$. (E-F) Correlation between serum sFasL and BAFF concentrations in (E) SLE $(n=118)$ and $(F)$ $\mathrm{HC}(\mathrm{n}=17)$. $(\mathrm{G}-\mathrm{H})$ Correlation between serum sFasL/sFas ratio and BAFF concentrations in $(G) \operatorname{SLE}(n=118)$ and $(H) H C$ $(n=17)$. In $A-H$, correlations were examined using Spearman's correlation rank test. BAFF, B cell-activating factor from the tumour necrosis factor family; $\mathrm{HC}$, healthy control; sFas, soluble Fas; sFasL, soluble Fas ligand; SLE, systemic lupus erythematosus.

GM $0.79,95 \%$ CI 0.58 to $1.07, \mathrm{p}=0.13$; mucocutaneous: adjusted for use of glucocorticoids and immunosuppressants: ratio of GM $0.89,95 \%$ CI 0.7 to $1.13, \mathrm{p}=0.35$ ). The sFasL/sFas ratio was also negatively associated with flare, confirmed after adjusting for use of immunosuppressants (ratio of GM $0.73 ; 95 \%$ CI 0.54 to $1 ; \mathrm{p}=0.05$ ), and with high ESR (table 2). We did not observe any significant association between $\mathrm{sFasL} / \mathrm{sFas}$ ratio and the presence of organ damage or other laboratory markers (table 2). 
Table 2 Univariable associations of serum sFas, sFasL, and sFasL/sFas ratio with SLE clinical parameters at baseline

\begin{tabular}{|c|c|c|c|c|c|c|c|c|}
\hline sFas & & & sFasL & & & sFasL/ & Fas ratio & \\
\hline $\begin{array}{l}\text { Ratio } \\
\text { of GM }\end{array}$ & (95\% Cl) & $P$ value & $\begin{array}{l}\text { Ratio } \\
\text { of GM }\end{array}$ & (95\% Cl) & $P$ value & $\begin{array}{l}\text { Ratio } \\
\text { of GM }\end{array}$ & (95\% Cl) & $P$ value \\
\hline
\end{tabular}

Clinical manifestations

Disease activity*

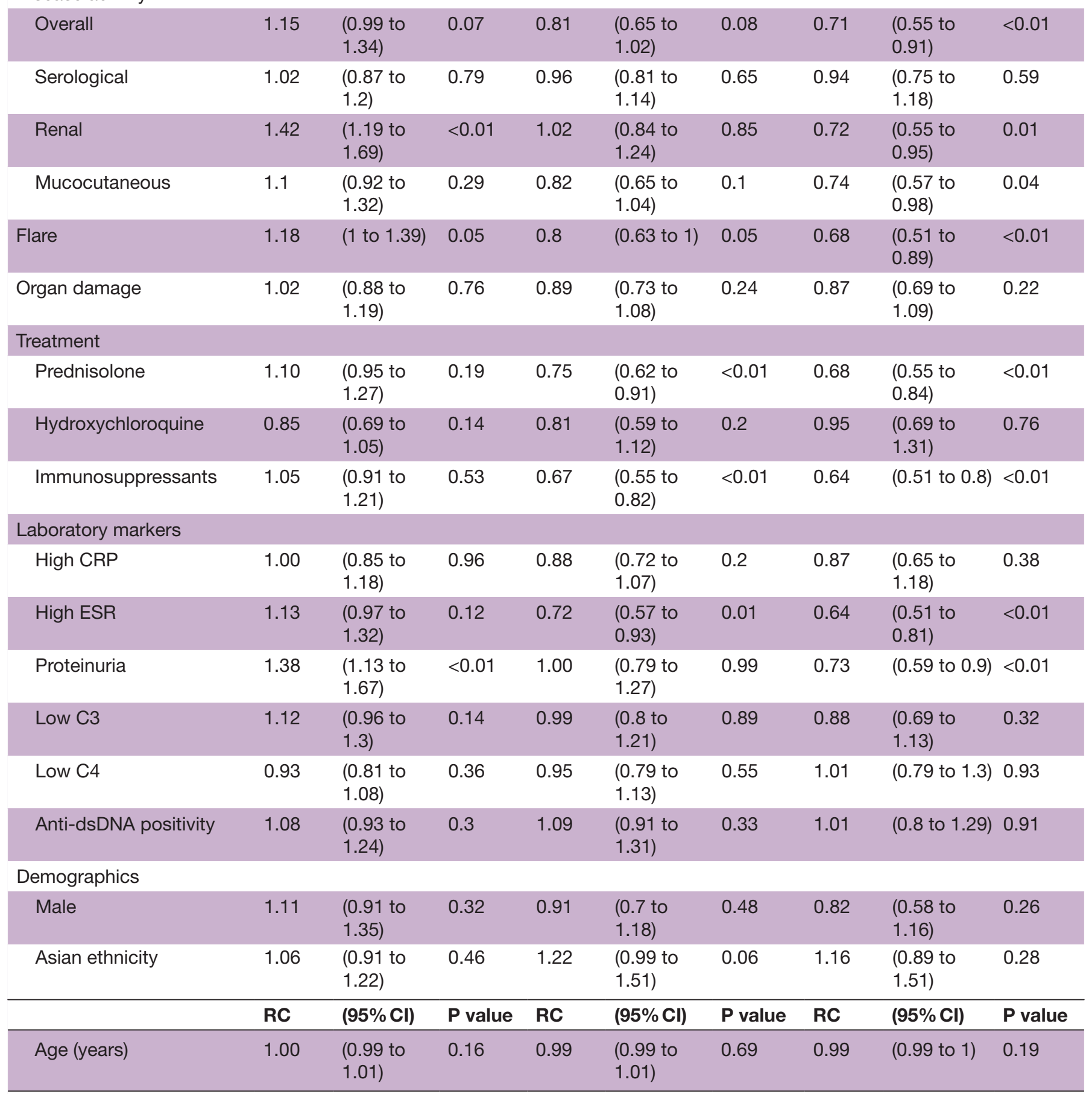

*Individual organ domain disease activity was assessed by SLEDAI-2K.

CRP, C reactive protein; dsDNA, double-stranded DNA; ESR, erythrocyte sedimentation rate; GM, geometric mean; RC, regression coefficient; sFas, soluble Fas; sFasL, soluble Fas ligand; SLEDAI-2K, Systemic Lupus Erythematosus Disease Activity Index 2000.

Clinical associations: longitudinal analysis

We next examined whether baseline serum concentrations were associated with clinical outcomes over time.
Patients with high baseline serum sFas were more than three times more likely to have active disease over time (AMS >4) (table 3), confirmed after adjusting for BAFF, 
Table 3 Univariable associations of baseline serum sFas, sFasL, and sFasL/sFas ratio with SLE clinical outcomes over time

\begin{tabular}{|c|c|c|c|c|c|c|c|c|c|c|c|c|}
\hline \multirow{2}{*}{$\begin{array}{l}\text { Baseline } \\
\text { serum sFas } \\
\text { and sFasL }\end{array}$} & \multicolumn{3}{|c|}{ AMS $>4$} & \multicolumn{3}{|c|}{ Flare over time } & \multicolumn{3}{|c|}{$\begin{array}{l}\text { Organ damage at last } \\
\text { visit }\end{array}$} & \multicolumn{3}{|c|}{ Damage accrual } \\
\hline & OR & $(95 \% \mathrm{Cl})$ & $P$ value & OR & $(95 \% \mathrm{Cl})$ & $P$ value & OR & $(95 \% \mathrm{Cl})$ & $P$ value & OR & $(95 \% \mathrm{Cl})$ & $\mathbf{P}$ value \\
\hline Low & 1.00 & & & 1.00 & & & 1.00 & & & 1.00 & & \\
\hline High & 3.01 & $\begin{array}{l}(1.39 \text { to } \\
6.52)\end{array}$ & $<0.01$ & 4.38 & $\begin{array}{l}(1.82 \text { to } \\
10.51)\end{array}$ & $<0.01$ & 1.67 & $\begin{array}{l}(0.75 \text { to } \\
3.71)\end{array}$ & 0.21 & 1.88 & $\begin{array}{l}(0.8 \text { to } \\
4.45)\end{array}$ & 0.15 \\
\hline \multicolumn{13}{|l|}{ sFasL } \\
\hline Low & 1.00 & & & 1.00 & & & 1.00 & & & 1.00 & & \\
\hline High & 0.86 & $\begin{array}{l}(0.41 \text { to } \\
1.8)\end{array}$ & 0.69 & 0.4 & $\begin{array}{l}(0.18 \text { to } \\
0.91)\end{array}$ & 0.03 & 0.3 & $\begin{array}{l}(0.13 \text { to } \\
0.69)\end{array}$ & $<0.01$ & 0.44 & $\begin{array}{l}(0.18 \text { to } \\
1.05)\end{array}$ & 0.06 \\
\hline \multicolumn{13}{|l|}{$\begin{array}{l}\text { sFasL/sFas } \\
\text { ratio }\end{array}$} \\
\hline
\end{tabular}

Low and high values were those below or equal to, or above, respectively, the median value for each cytokine.

AMS, time-adjusted mean Systemic Lupus Erythematosus Disease Activity Index 2000; sFas, soluble Fas; sFasL, soluble Fas ligand; SLE, systemic lupus erythematosus.

patients' age and use of glucocorticoids and immunosuppressants (OR 3.43; 95\% CI 1.32 to 8.9 ; $\mathrm{p}=0.01$ ). Patients with high baseline serum sFas were also more than four times likely to have flare of disease over time (table 3), confirmed after adjusting for use of glucocorticoids and immunosuppressants (OR 3.52; 95\% CI 1.4 to 8.86; $\mathrm{p}<0.01)$. No significant association was observed between serum sFas and damage accrual (table 3 ).

In contrast, patients with high baseline sFasL had a $60 \%$ reduction in flares over time and $70 \%$ reduction in risk of organ damage at the last visit (table 3). The association between sFasL and organ damage at the last visit remained significant after adjusting for ethnicity (OR 0.35 ; $95 \%$ CI 0.15 to $0.82 ; \mathrm{p}=0.02$ ), but the association with flare over time attenuated after adjusting for use of glucocorticoids and immunosuppressants (OR 0.57 ; 95\% CI 0.23 to 1.37 ; $\mathrm{p}=0.21$ ). Increased baseline sFasL was also negatively associated with damage accrual, with borderline significance (table 3). No significant association was observed between serum sFasL and AMS (table 3).

Examining serum sFasL/sFas ratio, univariable analysis revealed strong negative associations with flares over time and damage accrual (table 3). The association between sFasL/sFas ratio and organ damage at the last visit remained significant after adjusting for ethnicity (OR $0.41 ; 95 \%$ CI 0.17 to $0.94 ; \mathrm{p}=0.04$ ). The association between sFasL/sFas ratio and damage accrual remained significant after adjusting for use of glucocorticoids, a major driver of damage accrual in SLE (OR 0.35; 95\% CI 0.13 to $0.89 ; \mathrm{p}=0.03$ ). However, the association with flare over time attenuated after adjusting for use of glucocorticoids and immunosuppressants (OR 0.58; 95\% CI 0.24 to $1.4 ; \mathrm{p}=0.23)$. No significant association was observed between sFasL/sFas ratio and AMS (table 3). Including baseline organ damage in this multivariable model did not impact on the magnitude or significance of the association of sFasL/Fas ratio with damage accrual (OR 0.37; $95 \%$ CI 0.14 to $0.97 ; \mathrm{p}=0.04$ ).

\section{DISCUSSION}

The Fas/FasL system has a potential role in SLE pathogenesis. Here, we examined the clinical associations of sFas and sFasL in human SLE, and investigated whether their associations with SLE are dependent on any relationship with BAFF. We found that serum sFas was associated with active renal SLE, independent of serum BAFF. We also observed that serum sFasL was associated with organ damage accrual, also independent of serum BAFF. Serum sFasL/sFas ratio was associated with disease outcomes including disease activity, flare and organ damage accrual.

In this study, we confirmed previous reports of increased levels of serum sFas and sFasL in SLE compared with HC. ${ }^{10}{ }^{17-26} 29$ The sFasL/sFas ratio was not significantly different between SLE and HC after adjusting for age, in contrast to the findings of the sole previous study which investigated the use of this ratio in SLE. ${ }^{21}$ We report for the first time a positive relationship between serum BAFF and sFas in human SLE. The absence of statistically significant correlation between serum BAFF and sFas in $\mathrm{HC}$ may be explained by the modest HC sample size. Recent evidence in the lupus-prone BAFF-transgenic mouse model suggests a role for BAFF, a key SLE pathogenic cytokine ${ }^{36}$ in the regulation of the Fas/FasL apoptotic pathway, ${ }^{39}$ characterised by a defect in membrane-bound form of FasL upregulation. ${ }^{39}$ The positive relationship between serum BAFF and sFas underlines the relationship of the Fas/FasL and BAFF systems, and the requirement 
for BAFF to be adjusted for in clinical studies of the Fas/ FasL system, which should also be undertaken in autoimmune diseases other than SLE, such as primary Sjögren's syndrome.

We found a positive relationship between serum sFas and active SLE renal disease, in line with previous smaller studies. ${ }^{16} 182052$ We show that the association between serum $\mathrm{sFas}$ and renal disease activity was independent of BAFF, another renal SLE biomarker. ${ }^{53}$ Serum sFas may reflect not only its systemic production, but also local production in various organs, such as in situ kidney production, ${ }^{17}$ as well as being affected by kidney function. ${ }^{54}$ Although the present study cannot demonstrate any causal link, increased sFas decoy function could lead to increased immune cell survival. ${ }^{10}$ In the high serum sFas environment of autoimmune disease, the presence of oligomeric sFas ${ }^{1516}$ could lead to proapoptotic effects on mFasL-bearing resident renal cells. The assay used in the present study did not discriminate between monomeric and oligomeric sFas forms.

Serum sFas was also associated with flare and was a predictive biomarker for overall disease activity and flare over time, also independent of BAFF. This is in line with previous studies reporting sFas as a potential predictive marker for SLE relapse. ${ }^{405}$ We found no association of serum sFas with organ damage, in contrast to prior smaller cross-sectional studies ${ }^{17} 25$ and one which reported an association between serum sFas and organ damage longitudinally. ${ }^{19}$ While serum levels of BAFF and sFas were correlated, associations of sFas with clinical parameters were independent of BAFF. This suggests that, while an interplay between the BAFF/a proliferation-inducing ligand (APRIL) and Fas/FasL systems has been described in a lupus-prone mouse model ${ }^{39}$ BAFF may not be relevant to the association of sFas with SLE clinical outcomes.

Few published studies report on the relationship between serum sFasL and SLE disease activity, with most focusing on the relationship with overall activity, not phenotypic manifestations. ${ }^{26}{ }^{30}$ We report for the first time a negative relationship between serum sFasL and organ damage, where high baseline sFasL was associated with lower odds of organ damage. This suggests potential for sFasL as a protective biomarker. Serum BAFF was not a confounder for this association.

The use of a ratio between sFas and sFasL has been investigated in various conditions ${ }^{32-35}$; however, only one small study has assessed its clinical relevance in SLE. ${ }^{21}$ We observed clinical associations of serum sFasL/sFas ratio with overall SLE disease activity, as well as flare of disease. We also found an association between baseline $\mathrm{sFasL} / \mathrm{sFas}$ ratio and damage accrual over time. Overall, this study is the first showing significant clinical associations of sFasL/sFas ratio in SLE, particularly with organ damage accrual.

Caveats to the interpretation of the present data apply. First, although this study was conducted on a well-characterised SLE cohort, it was monocentric.
Second, the HC cohort was of modest sample size, although large enough to demonstrate a significant difference in sFas and sFasL between SLE and HC. Third, the HC cohort was not age-matched to the SLE cohort; however, age was included in multivariable regression to account for this difference. Finally, in common with many such studies, the number of SLE patients suffering from manifestations such as neurological, serosal or musculoskeletal disease was small, precluding any meaningful statistical analysis in these phenotypic subsets.

In conclusion, identifying relationships with organ disease may help understanding of the Fas/FasL system as targets for therapeutic intervention. We showed that sFas, sFasL and the ratio between them were associated with SLE disease outcomes. Serum sFas was particularly associated with active renal SLE, independently of BAFF, and may be a predictive biomarker for active disease and flare. In contrast, serum sFasL and the ratio between sFasL and sFas may be new biomarkers for protection from organ damage. Further research is needed to determine whether these soluble components may be valuable therapeutic targets in SLE, particularly in lupus nephritis and damage prevention.

Acknowledgements The authors wish to warmly thank the patients and healthy subjects for their participation in this study. They also thank the clinical staff of the Lupus Clinic at Monash Medical Centre, particularly Ms Sue Morton and Andrew Toh. The authors also thank the 360biolabs for assistance with the multiplex assays, in particular Ms Devy Santoso, and the French Society of Rheumatology for its support.

Contributors All authors contributed to experimental design, analysis and drafting of the paper. FV performed the ELISA and multiplex assays. Both FV and RK-R analysed the data. FV drafted the manuscript. All authors read and gave final approval of the version to be published.

Funding FV is a recipient of a Fellowship from Arthritis Australia. FM is a recipient of Fellowships from the NHMRC of Australia. EM was supported by the Kim Jolly Lupus Research Trust. The Monash Lupus database has received support from Arthritis and 0steoporosis Victoria, and unrestricted educational grants from GlaxoSmithKline, UCB, AstraZeneca and Eli Lilly Australia.

Competing interests EM has been a consultant to GSK and Eli Lilly. Other authors have no conflict of interest to declare.

Patient and public involvement Patients and/or the public were not involved in the design, or conduct, or reporting, or dissemination plans of this research. Patient consent for publication Not required.

Ethics approval This study was approved by the Human Research Ethics Committee, Monash Health. The study was carried out in accordance with the National Statement on Ethical Conduct in Human Research (2007).

Provenance and peer review Not commissioned; externally peer reviewed. Data availability statement Data are available upon reasonable request. Reasonable requests to view the data set used in this manuscript can be made in writing to FV (fabien.vincent@monash.edu).

Open access This is an open access article distributed in accordance with the Creative Commons Attribution Non Commercial (CC BY-NC 4.0) license, which permits others to distribute, remix, adapt, build upon this work noncommercially, and license their derivative works on different terms, provided the original work is properly cited, appropriate credit is given, any changes made indicated, and the use is non-commercial. See: http://creativecommons.org/ licenses/by-nc/4.0/.

\section{ORCID iDs}

Fabien B Vincent http://orcid.org/0000-0001-7220-0800

Alberta Y Hoi http://orcid.org/0000-0002-9416-7383 


\section{REFERENCES}

1 Lisnevskaia L, Murphy G, Isenberg D. Systemic lupus erythematosus. Lancet 2014;384:1878-88.

2 Liphaus BL, Kiss MHB. The role of apoptosis proteins and complement components in the etiopathogenesis of systemic lupus erythematosus. Clinics 2010;65:327-33.

3 Figgett WA, Vincent FB, Saulep-Easton D, et al. Roles of ligands from the TNF superfamily in B cell development, function, and regulation. Semin Immunol 2014;26:191-202.

4 Cohen PL, Eisenberg RA. Lpr and gld: single gene models of systemic autoimmunity and lymphoproliferative disease. Annu Rev Immunol 1991;9:243-69.

5 Watanabe-Fukunaga R, Brannan $\mathrm{Cl}$, Copeland NG, et al. Lymphoproliferation disorder in mice explained by defects in Fas antigen that mediates apoptosis. Nature 1992;356:314-7.

6 Takahashi T, Tanaka M, Brannan Cl, et al. Generalized lymphoproliferative disease in mice, caused by a point mutation in the Fas ligand. Cell 1994;76:969-76.

7 Jang S, Krammer PH, Salgame P. Lack of proapoptotic activity of soluble CD95 ligand is due to its failure to induce CD95 oligomers. $J$ Interferon Cytokine Res 2003;23:441-7.

8 Suda T, Hashimoto H, Tanaka M, et al. Membrane Fas ligand kills human peripheral blood T lymphocytes, and soluble Fas ligand blocks the killing. J Exp Med 1997;186:2045-50.

9 Hohlbaum AM, Moe S, Marshak-Rothstein A. Opposing effects of transmembrane and soluble Fas ligand expression on inflammation and tumor cell survival. J Exp Med 2000;191:1209-20.

10 Cheng J, Zhou T, Liu C, et al. Protection from Fas-mediated apoptosis by a soluble form of the Fas molecule. Science 1994;263:1759-62.

11 Cascino I, Fiucci G, Papoff G, et al. Three functional soluble forms of the human apoptosis-inducing Fas molecule are produced by alternative splicing. J Immunol 1995;154:2706-13.

12 O' Reilly LA, Tai L, Lee L, et al. Membrane-Bound Fas ligand only is essential for Fas-induced apoptosis. Nature 2009;461:659-63.

13 Matsumoto H, Murakami Y, Kataoka K, et al. Membrane-Bound and soluble Fas ligands have opposite functions in photoreceptor cell death following separation from the retinal pigment epithelium. Cell Death Dis 2015;6:e1986.

14 Audo R, Calmon-Hamaty F, Papon L, et al. Distinct effects of soluble and membrane-bound Fas ligand on fibroblast-like synoviocytes from rheumatoid arthritis patients. Arthritis Rheumatol 2014;66:3289-99.

15 Proussakova OV, Rabaya NA, Moshnikova AB, et al. Oligomerization of soluble Fas antigen induces its cytotoxicity. J Biol Chem 2003;278:36236-41.

16 Telegina E, Reshetnyak T, Moshnikova A, et al. A possible role of Fas-ligand-mediated "reverse signaling" in pathogenesis of rheumatoid arthritis and systemic lupus erythematosus. Immunol Lett 2009;122:12-17.

$17 \mathrm{Hao} \mathrm{JH}$, Ye DQ, Zhang GQ, et al. Elevated levels of serum soluble Fas are associated with organ and tissue damage in systemic lupus erythematosus among Chinese. Arch Dermatol Res 2006;297:329-32.

18 Sahebari M, Hatef MR, Rezaieyazdi Z, et al. Correlation between serum levels of soluble Fas (CD95/Apo-1) with disease activity in systemic lupus erythematosus patients in Khorasan, Iran. Arch Iran Med 2010;13:135-42.

19 Al-Maini MH, Mountz JD, Al-Mohri HA, et al. Serum levels of soluble Fas correlate with indices of organ damage in systemic lupus erythematosus. Lupus 2000;9:132-9.

20 Bollain-Y-Goytia JJ, Arellano-Rodríguez M, Torres-Del-Muro FdeJ, et al. Soluble Fas and the -670 polymorphism of Fas in lupus nephritis. Int J Nephrol 2014;2014:780406.

21 Turi MC, D'Urbano M, Celletti E, et al. Serum sFas/sFasL ratio in systemic lupus erythematosus (SLE) is a function of age. Arch Gerontol Geriatr 2009;49:221-6.

22 Jodo S, Kobayashi S, Kayagaki N, et al. Serum levels of soluble Fas/ APO-1 (CD95) and its molecular structure in patients with systemic lupus erythematosus (SLE) and other autoimmune diseases. Clin Exp Immunol 1997;107:89-95.

23 Araste JM, Sarvestani EK, Aflaki E, et al. Fas gene polymorphisms in systemic lupus erythematosus and serum levels of some apoptosisrelated molecules. Immunol Invest 2010;39:27-38.

24 Courtney PA, Crockard AD, Williamson K, et al. Lymphocyte apoptosis in systemic lupus erythematosus: relationships with Fas expression, serum soluble Fas and disease activity. Lupus 1999;8:508-13.

25 van der Linden MW, van Lopik T, Aarden LA, et al. Soluble CD95 concentrations are increased in patients with severe systemic lupus erythematosus, but not in their first degree relatives. Ann Rheum Dis 2001;60:237-41.
26 Tinazzi E, Puccetti A, Gerli R, et al. Serum DNase I, soluble Fas/ FasL levels and cell surface Fas expression in patients with SLE: a possible explanation for the lack of efficacy of hrDNase I treatment. Int Immunol 2009;21:237-43.

27 Fushimi M, Furukawa F, Tokura Y, et al. Membranous and soluble forms of Fas antigen in cutaneous lupus erythematosus. J Dermatol 1998;25:302-8.

28 Alecu M, Coman G, Alecu S. Serological levels of apoptotic bodies, sFAS and TNF in lupus erythematosus. Rom $\mathrm{J}$ Intern Med 2000;38:83-8.

29 Tauzin S, Chaigne-Delalande B, Selva E, et al. The naturally processed CD95L elicits a c-yes/calcium/PI3K-driven cell migration pathway. PLoS Biol 2011;9:e1001090.

30 Horák P, Scudla V, Hermanovó Z, et al. Clinical utility of selected disease activity markers in patients with systemic lupus erythematosus. Clin Rheumatol 2001;20:337-44.

31 Nadal C, Maurel J, Gallego R, et al. Fas/Fas ligand ratio: a marker of oxaliplatin-based intrinsic and acquired resistance in advanced colorectal cancer. Clin Cancer Res 2005;11:4770-4.

32 Adly AA, Ismail EA, Andrawes NG, et al. Soluble Fas/FasL ratio as a marker of vasculopathy in children and adolescents with sickle cell disease. Cytokine 2016;79:52-8.

33 Musiał K, Zwolińska D. Matrix metalloproteinases and soluble Fas/ FasL system as novel regulators of apoptosis in children and young adults on chronic dialysis. Apoptosis 2011;16:653-9.

34 Yildiz R, Benekli M, Buyukberber S, et al. The effect of bevacizumab on serum soluble Fas/FasL and TRAIL and its receptors (DR4 and DR5) in metastatic colorectal cancer. J Cancer Res Clin Oncol 2010;136:1471-6.

35 Vincent FB, Bubicich M, Downie-Doyle S, et al. Serum soluble Fas and Fas ligand (FasL) in primary Sjögren's syndrome. Clin Exp Rheumatol 2019;37 Suppl 118:254-6.

36 Vincent FB, Morand EF, Schneider P, et al. The BAFF/APRIL system in SLE pathogenesis. Nat Rev Rheumatol 2014;10:365-73.

37 Lemay S, Mao C, Singh AK. Cytokine gene expression in the MRL/lpr model of lupus nephritis. Kidney Int 1996;50:85-93.

38 Gross JA, Johnston J, Mudri S, et al. Taci and BCMA are receptors for a TNF homologue implicated in B-cell autoimmune disease. Nature 2000;404:995-9.

39 Figgett WA, Fairfax K, Vincent FB, et al. The TACI receptor regulates T-cell-independent marginal zone $B$ cell responses through innate activation-induced cell death. Immunity 2013;39:573-83.

40 Munroe ME, Vista ES, Guthridge JM, et al. Proinflammatory adaptive cytokine and shed tumor necrosis factor receptor levels are elevated preceding systemic lupus erythematosus disease flare. Arthritis Rheumatol 2014;66:1888-99.

41 Hochberg MC. Updating the American College of rheumatology revised criteria for the classification of systemic lupus erythematosus. Arthritis Rheum 1997;40:40.

42 Thanou A, Chakravarty E, James JA, et al. How should lupus flares be measured? deconstruction of the safety of estrogen in lupus erythematosus national assessment-systemic lupus erythematosus disease activity index flare index. Rheumatology 2014;53:2175-81.

43 Franklyn K, Lau CS, Navarra SV, et al. Definition and initial validation of a lupus low disease activity state (LLDAS). Ann Rheum Dis 2016;75:1615-21.

44 Mende R, Vincent FB, Kandane-Rathnayake R, et al. Analysis of Serum Interleukin (IL)-1 $\beta$ and IL-18 in Systemic Lupus Erythematosus. Front Immunol 2018;9:1250.

45 Ibañez D, Gladman DD, Urowitz MB. Adjusted mean systemic lupus erythematosus disease activity Index-2K is a predictor of outcome in SLE. J Rheumatol 2005;32:824-7.

46 Ibañez D, Urowitz MB, Gladman DD. Summarizing disease features over time: I. adjusted mean SLEDAI derivation and application to an index of disease activity in lupus. J Rheumatol 2003;30:1977-82.

47 Godsell J, Rudloff I, Kandane-Rathnayake R, et al. Clinical associations of IL-10 and IL-37 in systemic lupus erythematosus. Sci Rep 2016;6:34604.

48 Petri M, Kim MY, Kalunian KC, et al. Combined oral contraceptives in women with systemic lupus erythematosus. $N$ Engl J Med 2005;353:2550-8.

49 Gladman DD, Goldsmith $\mathrm{CH}$, Urowitz MB, et al. The systemic lupus international collaborating Clinics/American College of rheumatology (SLICC/ACR) damage index for systemic lupus erythematosus international comparison. J Rheumatol 2000;27:373-6.

50 Vincent FB, Nim HT, Lee JPW, et al. Effect of storage duration on cytokine stability in human serum and plasma. Cytokine 2019;113:453-7

51 Vincent FB, Kandane-Rathnayake R, Koelmeyer R, et al. Analysis of serum $B$ cell-activating factor from the tumor necrosis factor family 
(BAFF) and its soluble receptors in systemic lupus erythematosus. Clin Transl Immunology 2019;8:e01047:e1047.

52 Fathi NA, Hussein MR, Hassan HI, et al. Glomerular expression and elevated serum Bcl-2 and Fas proteins in lupus nephritis: preliminary findings. Clin Exp Immunol 2006;146:339-43.

53 Vincent FB, Northcott M, Hoi A, et al. Association of serum B cell activating factor from the tumour necrosis factor family (BAFF) and a proliferation-inducing ligand (April) with central nervous system and renal disease in systemic lupus erythematosus. Lupus 2013;22:873-84.

54 Christensson M, Pettersson E, Eneslätt K, et al. Serum sFAS levels are elevated in ANCA-positive vasculitis compared with other autoimmune diseases. J Clin Immunol 2002;22:220-7.

55 van Lopik T, Bijl M, Hart M, et al. Patients with systemic lupus erythematosus with high plasma levels of sFas risk relapse. $J$ Rheumatol 1999;26:60-7. 